\title{
Isolation and expression analysis of four HD-ZIP III family genes targeted by microRNA166 in peach
}

\author{
C.H. Zhang, B.B. Zhang, R.J. Ma, M.L. Yu, S.L. Guo and L. Guo \\ Institute of Horticulture, Jiangsu Academy of Agricultural Sciences/Jiangsu Key \\ Laboratory for Horticultural Crop Genetic Improvement, Nanjing, Jiangsu, China \\ Corresponding author: R.J. Ma \\ E-mail: marj311@163.com \\ Genet. Mol. Res. 14 (4): 14151-14161 (2015) \\ Received July 3, 2015 \\ Accepted September 18, 2015 \\ Published October 29, 2015 \\ DOI http://dx.doi.org/10.4238/2015.October.29.37
}

\begin{abstract}
MicroRNA166 (miR166) is known to have highly conserved targets that encode proteins of the class III homeodomain-leucine zipper (HD-ZIP III) family, in a broad range of plant species. To further understand the relationship between HD-ZIP III genes and miR166, four HD-ZIP III family genes (PpHB14, PpHB15, PpHB8, and PpREV) were isolated from peach (Prunus persica) tissue and characterized. Spatio-temporal expression profiles of the genes were analyzed. Genes of the peach HDZIP III family were predicted to encode five conserved domains. Deduced amino acid sequences and tertiary structures of the four peach HD-ZIP III genes were highly conserved, with corresponding genes in Arabidopsis thaliana. The expression level of four targets displayed the opposite trend to that of miR166 throughout fruit development, with the exception of PpHB14 from 35 to 55 days after full bloom (DAFB). This finding indicates that miR166 may negatively regulate its four targets throughout fruit development. As for leaf and phloem, the same trend in expression level was observed between four targets and miR166 from 75 to 105 DAFB. However, the opposite trend was observed for the transcript level between four targets and miR166 from 35 to 55 DAFB. miRNA166 may negatively
\end{abstract}


regulate four targets in some but not all developmental stages for a given tissue. The four genes studied were observed to have, exactly or generally, the same change tendency as individual tissue development, a finding that suggests genes of the HD-ZIP III family in peach may have complementary or cooperative functions in various tissues.

Key words: Peach; MicroRNA166; HD-ZIP III family genes; Isolation; Gene expression

\section{INTRODUCTION}

MicroRNAs (miRNAs) are single-stranded noncoding RNAs ranging in size from approximately 20-22 nucleotides (nt), which post-transcriptionally and negatively regulate the expression of target genes in plants. Since the discovery of the first miRNA in plants in 2002 (Park et al., 2002), thousands of miRNAs have been identified experimentally or computationally from a variety of species, such as beans (Arenas-Huertero et al., 2009), peanut (Zhao et al., 2010), maize (Jiao et al., 2011), and opium poppy (Unver et al., 2010). Many miRNA families are evolutionarily conserved across plant species, in which they are capable of regulating expression of protein-coding genes (Asha et al., 2013). Plant miRNAs are highly complementary to their targets, with no more than fournt mismatches (Bartel, 2004). They are known to play crucial roles in various processes (Eldem et al., 2013) and functions in plants, such as tissue morphogenesis (Palatnik et al., 2003); embryonic organ formation and separation (Palatnik et al., 2003); floral development (Mallory et al., 2004); and stress response (Jones-Rhoades and Bartel, 2004; Chen, 2004). They regulate the functions of their target genes by binding to complementary sequences in target mRNAs, resulting in transcript cleavage (Zhong et al., 2007). Accumulation of various plant miRNAs differs throughout plant developmental stages, suggesting that their expression may be spatially and temporally regulated.

MicroRNAs that are conserved in plants also tend to have conserved targets, and when a miRNA targets multiple mRNAs, the targeted genes are often members of the same gene family (Rhoades et al., 2002; Jones-Rhoades and Bartel, 2004). The conserved miR166 family is widely considered to have highly conserved targets that encode proteins of the class III homeodomainleucine zipper (HD-ZIP III) family in a broad range of plant species that includes olive (Donaire et al., 2011), citrus (Song et al., 2009), pepper (Asha et al., 2013), and cotton (Qiu et al., 2007). Members of the HD-ZIP III transcriptional factors are plant-specific and regulate critical aspects of plant development, including lateral organ polarity, apical and lateral meristem formation, and vascular development (Ruberti et al., 1991). The HD-ZIP III family comprises five genes in Arabidopsis thaliana: ATHB8; phavoluta ( $P H V$, also called ATHB9); phabulosa ( $P H B$, also called ATHB14); revoluta $[R E V$, also called inter fascicular fiberless 1, or (IFL1)]; and corona (CNA, also called ATHB15 or incurvata4 (ICU4)) (Prigge et al., 2005). The apparent actions of HD-ZIP III proteins such as PHB, PHV, REV, ATHB8, and ATHB15 (CNAMCU4), are to decide and direct differentiation versus maintenance in cells of the shoot apical meristem (Emery et al., 2003; Prigge and Clark, 2006). It is reported that the expression of Arabidopsis thaliana REV is closely associated with wood formation, and is regulated developmentally and seasonally (Ko et al., 2006). MicroRNA166 is able to regulate functions of target genes by binding to complementary sequences in the target transcripts of HD-ZIP III genes to cause transcript cleavage. The transcript level of HD-ZIP III genes is therefore inversely correlated with that of miR166 (Rhoades et al., 2002). 
Peach (Prunus persica (L.) Batsch) is a popular fruit worldwide because of its high nutrient level and pleasant flavor. In the peach genome, a number of miRNAs have been identified, through sequencing of small RNAs combined with computational prediction. Among them, miR166 is predicted to target the four genes of HD-ZIP III family, and has four nucleotide mismatches in the complementary area with three target genes (gene ID ppa001378m, ppa001343m, ppa001386m for CDS downloaded from GDR website) and three mismatches with one target gene (gene ID ppa001405m), respectively. Combined with our previous study on HD-ZIP family (Zhang et al., 2014) and NCBI BLAST using nucleotide sequences of HD-ZIP III family genes in Arabidopsis thaliana, peach genes with ID ppa001343m, ppa001405m, ppa001386m, and ppa001378m were named PpHB14, PpHB15, PpHB8, and PpREV in this work, respectively. Gene ontology (GO) annotation indicated the four genes function in nucleic acid binding and take part in many processes including meristem structural organization, anatomical structure arrangement, regulation of cell differentiation, and reproductive structure development. One question that remains is whether miR166 plays differential roles in the regulation of the HD-ZIP III genes in different tissues.

Efforts have been made to predict and identify a large number of miRNAs and target genes in various tissues of peach. However, little information is available for further analysis of miRNAs and their targets in peach. For these reasons, the current study focused primarily on the isolation of four target genes encoding PpHB14, PpHB15, PpHB8, and PpREV proteins; and the expression analysis of miR166 and its targets $P p H B 14, P p H B 15, P p H B 8$, and PpREV at different developmental stages in three peach tissues.

\section{MATERIAL AND METHODS}

\section{Plant material}

Peach 'XiaHui8' ('XH8') growing at the National Peach Germplasm Repository in Nanjing, China (Institute of Horticulture, Jiangsu Academy of Agricultural Sciences) was used to source tissue samples. Peach trees used in the study were 7 years old and were all grown under the same field conditions and management. The peach orchard was divided into three plots, from which three trees per plot were selected for tissue collection. Fruits, phloem, and leaves from the same node were sampled from the outer southern canopies of trees at certain day intervals, from 35 days after full bloom (DAFB) through to fruit ripening. The fifth leaf from the apex of one-year-old fruiting shoots was picked at each sampling time. Phloem above and below the node of this leaf was peeled while fruits from the node of the sampled leaves were picked and chopped into pieces following stone removal. Tissue from each individual sampling point and date was mixed, placed in $50-\mathrm{mL}$ tubes, immediately frozen in liquid nitrogen, and stored at $-70^{\circ} \mathrm{C}$ until further use.

\section{RNA extraction, DNase treatment, and cDNA synthesis}

Total RNA was extracted from tissues using Trizol reagent (Invitrogen Corp., Carlsbad, CA, USA). Thereafter, RNA was treated with DNase I and reverse transcribed using a Prime-Script RT reagent kit with gDNA Eraser following the manufacturer instructions (Takara Biotechnology Co. Ltd, Dalian, Shenyang, China). All cDNA samples were diluted 1:10 in RNase-free water and verified by semi-quantitative reverse transcription polymerase chain reaction (RT-PCR). All cDNA was stored at $-20^{\circ} \mathrm{C}$ before use as a template in cloning and in quantitative realtime PCR (qPCR) for target mRNA. 
As for qPCR analysis of miR166 expression levels, small RNA (sRNA) was extracted from fruit, phloem and leaf tissues at different stages of peach development using an extraction kit (Takara Biotechnology), then treated with DNase I and reverse transcribed using a TransScript miRNA first-strand cDNA synthesis SuperMix kit, following the manufacturer instructions (TransGen Biotech Co., Beijing, China). All cDNA samples were diluted 1:10 in RNase-free water and stored at $-20^{\circ} \mathrm{C}$ before use as a template in qPCR for miR166.

\section{Cloning of HD-ZIP III subfamily genes}

Only cDNA reverse transcribed from fruit was used as template for the initial cloning of $P p H B 15, P p H B 14, P p H B 8$, and PpREV. Using the corresponding full-length cDNA sequences of each of the genes in peach predicted by Zhang et al. (2014), specific primers were designed using Primer Premier 5.0 software (Premier Biosoft, Canada), and synthesized by Invitrogen Biotechnology Co., Ltd. Primers are listed in Table 1.

\begin{tabular}{|c|c|c|c|}
\hline Gene name & Sequence $\left(5^{\prime}-3^{\prime}\right)$ & Size of amplified product (bp) & $\operatorname{Tm}\left({ }^{\circ} \mathrm{C}\right)$ \\
\hline PpHB14 & $\begin{array}{l}\text { ATGGCACTTGTTATGAACAGAGATT } \\
\text { TCACACAAAGGACCAGCTCATGAAC }\end{array}$ & 2550 & 55.1 \\
\hline PpHB15 & $\begin{array}{l}\text { ATGGCTATGTCCTGCAAGGAT } \\
\text { TCAGACGAAAGACCAGTTTACAAAT }\end{array}$ & 2514 & 55.1 \\
\hline PpREV & $\begin{array}{l}\text { ATGGCTATGGCTGTGGCTCA } \\
\text { TCACACAAAAGACCAGTTCATGAAG }\end{array}$ & 2529 & 55.1 \\
\hline РpHB8 & $\begin{array}{l}\text { ATGATGGCGGTGACGTCAGCC } \\
\text { TCAGACAAATGACCAGTTGAT }\end{array}$ & 2523 & 58.0 \\
\hline
\end{tabular}

Amplifications by PCR were performed in $25-\mu \mathrm{L}$ volumes containing: $2.5 \mu \mathrm{L} 10 \mathrm{X}$ PCR buffer; $0.5 \mu \mathrm{L} 10 \mathrm{mM}$ dNTP mixture, $2 \mu \mathrm{L} \mathrm{MgCl}_{2} ; 0.3 \mu \mathrm{L} \mathrm{LA}$ Taq polymerase (5U/ $\mu \mathrm{L}$; Takara Biotechnology); $2 \mu \mathrm{L}$ cDNA; $0.5 \mu \mathrm{L}$ of each gene-specific primer; and 16.7 $\mu \mathrm{L}$ RNase-free water. Amplification was performed in an Eppendorf authorized thermal cycler (Eppendorf Vertrieb Deutschland GmbH, Hamburg, Germany) using the following program: $94^{\circ} \mathrm{C}$ pre-denaturing for $5 \mathrm{~min}$; 34 cycles of $94^{\circ} \mathrm{C}$ for $1 \mathrm{~min}$, the primer-specific melting temperature (Tm) for $1 \mathrm{~min}$ (shown in Table 1), and $72^{\circ} \mathrm{C}$ for $1 \mathrm{~min}$; and $72^{\circ} \mathrm{C}$ for $10 \mathrm{~min}$. Amplified products were separated by electrophoresis on a $1-2 \%$ agarose $(\mathrm{w} / \mathrm{v}) \mathrm{gel}$, and visualized by staining with ethidium bromide. The target DNA fragment was purified using an Axyprep DNA gel extraction kit (Axygen Biosciences Co. Ltd., Hangzhou, Zhejiang, China), then ligated into Peasy-T3 vector and finally transformed into DH5a. Positive clones were identified using PCR analysis and sequenced by Invitrogen Biotechnology.

The similarity between HD-ZIP III genes in peach and other plants was determined using NCBI BLAST. Obtained CDNA sequences were further blasted to verify the open reading frame (ORF) of the predicted cDNA sequences, and BioXM (version 2.6, Institute of Rice in Nanjing Agricultural University, China) used to deduce amino acid (aa) sequences of the four genes. Deduced aa sequences of the four HD-ZIP III genes in peach, and corresponding genes in Arabidopsis, were imported to DNAMAN software (LynnonBiosoft, USA) to do a multiple alignment. In order to determine the domain distribution in each member of HD-ZIP III family proteins, the four protein sequences were searched for specific conserved domains using the InterProScan (http://www.ebi.ac.uk/Tools/ InterProScan/) and NCBI conserved domains (CD) search. Tertiary structure analysis was performed by homology modeling, using the online server ExPaSy Swiss-Model (http://swissmodel.expasy.org). 


\section{Primers for qPCR}

Gene-specific primers for qPCR amplification of the four target genes were designed based on the three cloned sequences above, using Primer Premier 5.0 software (Premier Biosoft, Canada). Amplicon lengths were optimized to $100-200 \mathrm{bp}$ to ensure optimal polymerization efficiency and minimize the effect of RNA integrity on the relative quantification of gene expression. Furthermore, one of the primers was optimized to be located within its 3 -untranslated region (UTR) to ensure primer specificity. Primers were further used to query the predicted peach genome database with Blastn, to confirm gene identities. Prior to $\mathrm{qPCR}$, each primer pair was tested via standard RT-PCR and electrophoresis onethidium bromide-stained $2 \%$ agarose $(\mathrm{w} / \mathrm{v})$ gels to verify size specificity of the amplified products. Amplified bands were cloned and sequenced to confirm they were indeed fragments of target genes. Translation elongation factor 2 (TEF2, TC3544) was used as a reference gene, based on a study by Tong et al. (2009), and following guidelines published by Bustin et al. (2009). Primer sequences, sizes of the amplified products, and optimal Tm for qPCR of each gene are listed in Table 2.

Table 2. Primer designed for qPCR of HD-ZIP III family genes and miR166 in peach.

\begin{tabular}{|c|c|c|c|}
\hline Gene name & Sequence $\left(5^{\prime}-3^{\prime}\right)$ & Size of amplified product (bp) & $\operatorname{Tm}\left({ }^{\circ} \mathrm{C}\right)$ \\
\hline \multirow[t]{2}{*}{ PpHB14 } & GGAGACCTTGGCAGAGTT & 184 & 60.0 \\
\hline & GAGGATTTCAGCGACCTT & & \\
\hline \multirow{2}{*}{ PpHB15 } & GACTATGGCGGCTTTACG & 107 & 60.0 \\
\hline & TCTGGCTTAGTGCTCGTA & & \\
\hline \multirow[t]{2}{*}{ PpREV } & TCAGAACCGAAGGTGTAGG & 186 & 60.0 \\
\hline & TCAGTAGTTGGTGCAGTATG & & \\
\hline \multirow[t]{2}{*}{ PpHB8 } & CTGCTGCTATTAAACCTG & 116 & 60.0 \\
\hline & GACCTCCATGAACTCTTC & & \\
\hline \multirow[t]{2}{*}{ TEF2 } & GGTGTGACGATGAAGAGTGATG & 129 & 60.0 \\
\hline & TGAAGGAGAGGGAAGGTGAAAG & & \\
\hline \multirow[t]{2}{*}{ 5S rRNA } & CTCGGCAACGGATATCTCGGCTCT & & \\
\hline & CTAATGGCTTGGGGCGCAACTTG & & \\
\hline $\operatorname{miR} 166$ & TCGGACCAGGCTTCATTCCCC & & \\
\hline
\end{tabular}

For expression analysis of miR 166 by qPCR, one specific primer was the mature sequence of miR166 and one was the universal primer from the TransScript kit. Ribosomal RNA (rRNA) $5 S$ was used as a reference gene.

\section{Quantitative PCR with SYBR green}

Reactions were conducted on a 7500 real-time PCR system (Applied Biosystems) using a TransStart top green qRT-PCR SuperMix according to the manufacturer instructions (TransGen Biotech). Reaction volumes were $20 \mu \mathrm{L}$ and contained $2 \mu \mathrm{L}$ diluted cDNA; $0.4 \mu \mathrm{L}$ primer; $0.4 \mu \mathrm{L}$ passive reference dye; $10 \mu \mathrm{L}$ supermix; and $6.8 \mu \mathrm{L}$ RNase-free water. Thermal cycling conditions included an initial polymerase activation step for $30 \mathrm{~s}$ at $95^{\circ} \mathrm{C}$ followed by 40 cycles of $5 \mathrm{~s}$ at $95^{\circ} \mathrm{C}$ for template denaturation, and $34 \mathrm{~s}$ at $60^{\circ} \mathrm{C}$ for annealing (fluorescence measurement). Stage 3 was set for $15 \mathrm{~s}$ at $95^{\circ} \mathrm{C}$, followed by $1 \mathrm{~min}$ at $60^{\circ} \mathrm{C}$ and $15 \mathrm{~s}$ at $95^{\circ} \mathrm{C}$. Each assay was replicated three times. Raw fluorescence data were exported to Microsoft Excel (Microsoft Corporation, USA), and relative quantification gene expression levels analyzed using the $2{ }^{-} \Delta \Delta \mathrm{Ct}$ method (Livak and Schmittgen, 2001). 


\section{RESULTS}

\section{Cloning of HD-ZIP III family genes}

Cloning led to the isolation of cDNAs of 2514, 2550, 2523, and 2529 nt in length, with full length ORFs encoding 837, 849, 840, and 842 aa for PpHB15, PpHB14, PpHB8, and PpREV, respectively, in peach. GenBank accession numbers for sequences are shown in Table 3. Because the nt sequence of $P p H B 8$ was identical to that previously isolated from another peach cultivar (Zhang et al., 2012), this sequence was not submitted to GenBank.

Table 3. Basic information of HD-ZIP III family genes isolated from peach.
\begin{tabular}{lcccc}
\hline Gene name & GenBank accession No. & Gene ID & ORF length & Amino acid No. \\
\hline PpHB14 & KJ000552 & Ppa001343m & 2550 & 849 \\
PpHB15 & KJ000553 & Ppa001405m & 2514 & 837 \\
PpREV & KJ000554 & Ppa001378m & 2529 & 842 \\
PpHB8 & JN865251 & Ppa001386m & 2523 & 840 \\
\hline
\end{tabular}

BLAST searching for PpHB14 nt sequence against the NCBI database revealed the highest identity $(87 \%)$ of the sequence obtained in our study with a HD-ZIP III protein mRNA of Betula luminifera (accession No. KJ451560.1), and 86\% identity with a PHABULOSA family protein mRNA in Populus trichocarpa (accession No. XM_002298856.2). PpHB15 shared 93\% homology with the HB15 protein mRNA of Malus domestica (accession No. NM_001293969.1), and 86\% homology with Populus alba x Populus glandulosa (accession No. KF534716.1). PpHB8 shared 91 and $85 \%$ nt sequence identity with HB8 of Malus domestica (accession No. NM_001293821.1) and Theobroma cacao (accession No. XM_007012090.1), respectively. PpREV had a similarity of 83\% with both REV mRNA in Malus $\mathrm{x}$ domestica (accession No. FJ177425.1) and Populus trichocarpa HD-ZIP III family protein mRNA (accession No. XM_006378911.1). Multiple alignments of deduced aa sequences of the four HD-ZIP III genes in peach and corresponding genes in Arabidopsis, demonstrated that the four HD-ZIP III proteins were highly conserved (Figure 1).

Four genes of peach HD-ZIP III family were predicted to encode a homeoboxdomain, a single START domain, a START-like domain, and a MEKHLA domain. The homeobox domain, the shortest of four domains in each protein, was approximately 68 to 76 aa in length, and was near the $5^{\prime}$ end of each protein. The MEKHLA domain is found atthe $3^{\prime}$ end of each protein encoded by thepeach HD-ZIP III family genes and ends with the last amino acid of each protein. The results obtained from InterProScan tools indicated the first domain located at residues 16 to 84 (PpHB8); 8 to 84 (PpHB14); 11 to 79 (PpHB15); and 22 to 88 (PpREV; Figure 2). The START domain was observed at residues 155 to 383 (PpHB8); 160 to 388 (PpHB14); 159 to 365 (PpHB15); and 152 to 380 (PpREV). Results from the CD search within proteins of the peach HD-ZIP III family showed that each member contained a homeoboxdomain, a leucinezipper domain, and a START ARGLABRA2 like domain, as well as a MEKHLA domain. Based on previouslypublished findings and results obtained in the current study, we concluded that the proteins of the peach HD-ZIP III family were characterized by their encoding of homeobox, leucine zipper, START, START-like, and MEKHLA domains. Although there were differences in the length of each domain among thepeach HD-ZIP III genes, a high level of conservation in domain organization and position could be observed, which was similar to observations from multiple alignment among the four aa sequences (shown in Figures 1 and 2). 


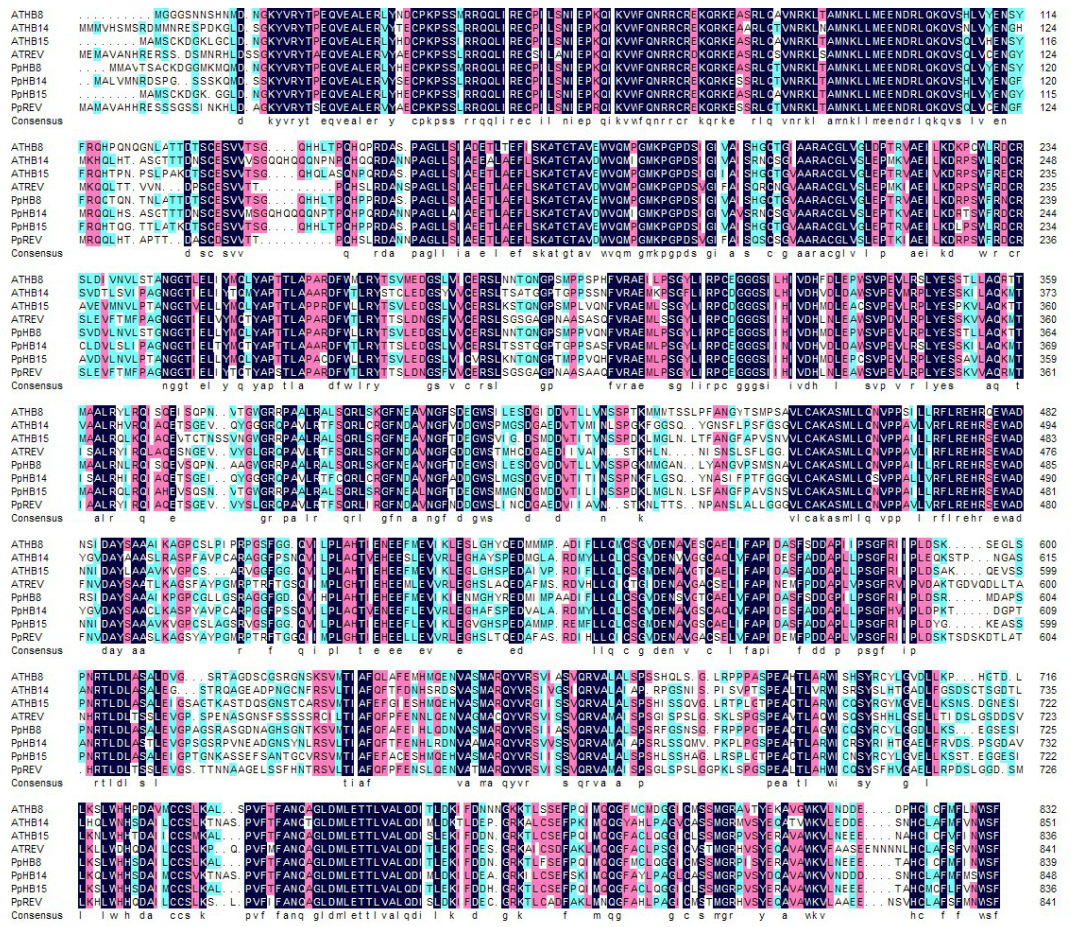

Figure 1. Alignment analysis of predicted amino acid sequences of the four HD-ZIP III family proteins in peach and Arabidopsis thaliana. Sequence alignment analysis was performed using the multiple alignment program of DNAMAN 6.0 software. Identical amino acids are shaded; gaps are indicated by dots.
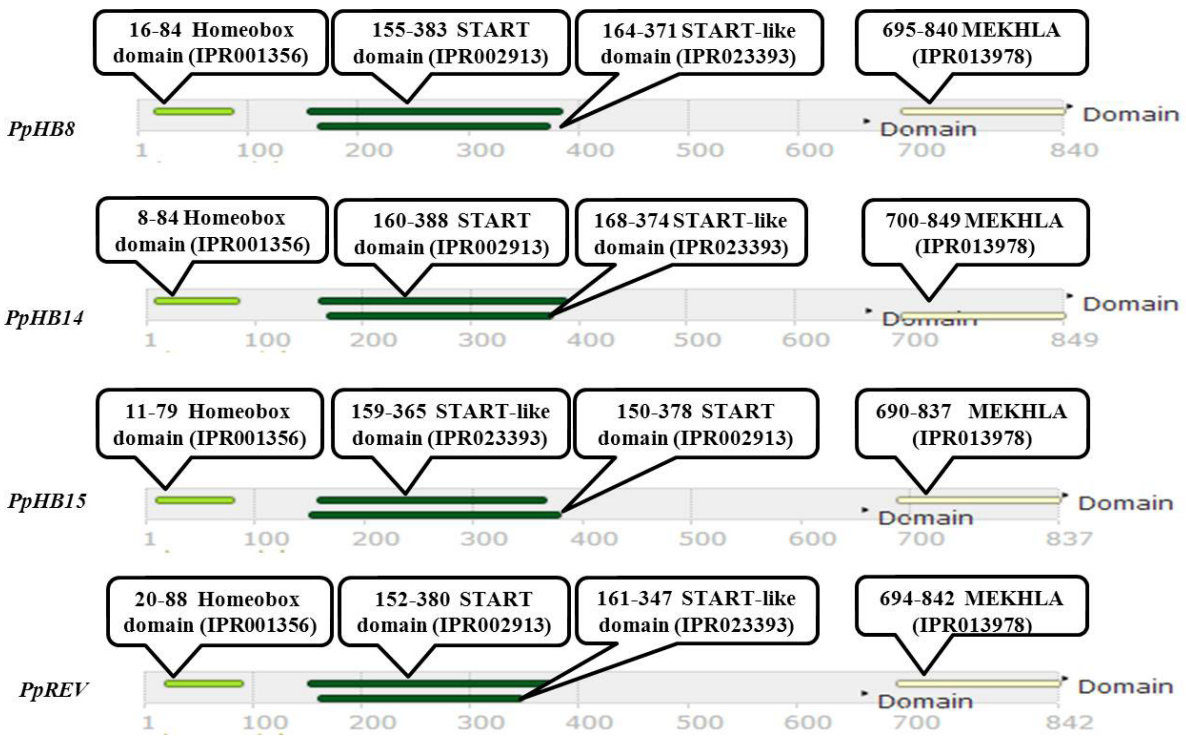

Figure 2. Domain distribution of HD-ZIP III family proteins in peach. Domains were investigated by InterPro server. Numbers depict amino acid location. 
Tertiary structures of predicted HD-ZIP III family proteins in peach are shown in Figure 3. The four proteins were found to have similar tertiary structures, but had slight differences in the length and aa composition of units that formed these tertiary structures.

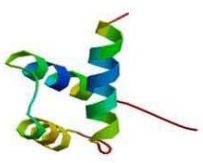

PрHB14

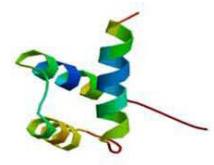

PpHB15

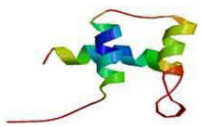

PpREV

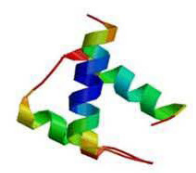

PpHB8

Figure 3. Tertiary structures of HD-ZIP III family proteins in peach. Analysis was performed using the online server ExPaSy Swiss-Model, which automatically causes amino acid sequences to form tertiary protein structures through homology-modeling tertiary structures. Estimated per-residue inaccuracy is visualized using a color gradient from blue (more reliable regions) to red (potentially unreliable region).

\section{Expression analysis of HD-ZIP III family genes}

The expression level of miR166 in fruit increased gradually from 35 to 90 DAFB (Figure 4) and reached the highest level (15-fold increase compared with 35 DAFB) at90 DAFB. A significant decrease in the expression of this gene was subsequently observed from 90 to 105 DAFB, and a very weak expression level was detected on the day of harvest. In general, the expression level of the four targets displayed opposite change trend compared with the expression level of miR166 throughout fruit development, with an exception of PpHB14 from 35 to 55 DAFB. The expression level of $P p H B 14$ had the same increased trend as miR166 from 35 to 55 DAFB.

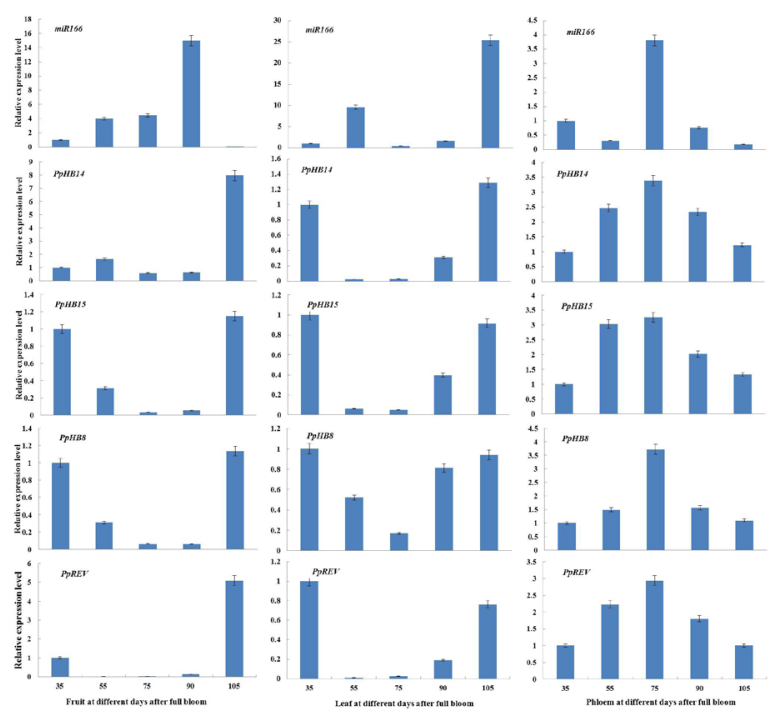

Figure 4. Expression analysis of genes encoding HD-ZIP III family proteins and miR166 in three tissues throughout peach development TEF2 and 5S rRNA were chosen as reference genes for the HD-ZIP family genes and miR166, respectively. Relative expression levels of each gene were detected in peach fruit, phloem, and leaf at $35,55,75,90$, and 105 days after full bloom (DAFB). Relative expression levels of each gene were calculated by the $2^{-\Delta \Delta C t}$ method. The $y$-axis shows the fold difference in each gene expression level relative to the amount found in each tissue sampled at different DAFB. Vertical bars represent \pm SEM $(\mathrm{N}=9)$. 
In leaf, there was a significant increase in the transcript level of all four targets and of miR166 from 75 to 105 DAFB. However, the opposite trend was observed from 35 to 55 DAFB. The four targets on the 35 and 105 DAFB showed higher expression levels than during other development periods. All targets as well as miR166 exhibited the same trend in phloem from 75 to 105 DAFB, but opposite change tendency from 35 to 55 DAFB.

In general, expression levels of $P p H B 14, P p H B 15, P p H B 8$, and $P p R E V$ had similar trends in fruit and leaf and these were substantially different from that observed for phloem. However, the four target genes were observed to have generally the same change tendency as leaf or phloem development in this study. The four HD-ZIP III family genes displayed high abundance in mature fruits and both leaves on 35 and 105 DAFB, respectively, as well as in phloem on 75 DAFB.

\section{DISCUSSION}

The identification of miRNA targets is an essential step toward understanding their regulatory function. The first plant genes identified to be under miRNA regulation were HD-ZIP III transcription factors (Turner et al., 2007). In this study, four targets (PpHB14, PpHB15, PpHB8, and PpREV) were identified in peach, based on previous high throughput sequencing and bioinformatics predictions for peach SRNA library. Domain analysis results showed each putative protein contained five domains; namely, homeobox, leucine zipper, START, START-like, and MEKHLA domains, which is mostly consistent with previous reports (Mukherjee and Burglin, 2006; Ariel et al., 2007; Javelle et al., 2011). However, it is somewhat different from the observation that HD-ZIP III family genes encode proteins with homeobox, leucine zipper dimerization, putative sterol-binding, and MEKHLA domains (Prigge and Clark, 2006). This may be due to differences in software used in those reports, or to differences between plant species. Furthermore, in the current study, the distribution order of the predicted domains in each protein sequence was the same as described in previous reports (Mukherjee and Burglin, 2006; Ariel et al., 2007). We verified here the four isolated HD-ZIP III mRNAs from peach share high similarity with those of Arabidopsis at the protein level. Consistent with previous reports on HD-ZIP III family members in other plants (Prigge and Clark, 2006; Sessa et al., 1998), the high similarity at protein level among four HD-ZIP III family members in peach is also observed in this study.

To shed light on the role of each HD-ZIP III transcription factor, we analyzed their expression level in three peach tissues at different developmental stages. The four HD-ZIP III transcription factors were abundantly expressed in mature fruit and leaves on 35 and 105 DAFB, as well as in phloem on 75 DAFB. Ong and Wickneswari (2012) found that four HD-ZIP III transcription factors were absent in the leaf tissues of Acacia mangium, based on results obtained from a single sampling time that did not examine expression levels at different developmental stages. In agreement with this partially, expression levels of $P p H B 14, P p H B 15$, and PpREV in leaves from 55 to 75 DAFB were very low/almost undetectable. These findings indicate that the function or effects of HD-ZIP III transcription factors on development may differ or change as leaves develop.

Characterizing expression of miRNAs in different tissues may provide clues as to the sites of function of these miRNAs (Asha et al., 2013). To improve our understanding of the complex regulatory network in various tissues in peach, we used qPCR technology to track changes in miR166 expression, in the same three tissues at the same five development stages. As both miR166 and its corresponding target sites are highly conserved across a wide range of species (Arenas-Huertero et al., 2009; Barik et al., 2014), information on the homologous miRNA and mRNA dataset will provide useful information on conserved regulatory roles in different species. Opposite expression patterns of the miR166 to its targets in different tissues at certain developmental stages 
suggested miR166 as the potential regulatory sequence in fruit, phloem, and leaf tissues.

Expression data showed that throughout fruit development, miR166 had opposite change tendency compared to its four target genes, indicating a negative regulatory function for miR166. However, the target genes and miR166 displayed the same change trend in leaf and phloem from 75 to 105 DAFB, but an opposite change tendency from 35 to 55 DAFB, indicating that miR166 in leaf and phloem may negatively regulate target genes at specific time points only. In agreement with findings by Ong and Wickneswari (2012) in xylem, our study suggests that miR166 may play a potential role in phloem primary development, as strong down regulation of miR166 in phloem from 35 to 55 DAFB was accompanied by up regulation of the four target genes. In agreement with previous reports (Rhoadeset al., 2002; Nicolas et al., 2008), our findings are a complementary identification that negative regulation of HD-ZIP III by miR166 is highly conserved in plant species. This may be because binding sites of miR166 are conserved in all HD-ZIP III family genes across a wide range of plant species (Floyd and Bowman, 2004). Moreover, we propose that miR166 may play different roles in different tissues, and may negatively regulate its four target genes in some but not all developmental stages for a given tissue. Namely, miR166 just has regulation function to its targets in some developmental periods of a tissue. Hence, miR166 is suggested to play a critical regulatory role in controlling gene expression of HD-ZIP III family in the fruit, leaf, and phloem in peach.

The four target genes of peach HD-ZIP III family were observed to have exactly or generally the same tendency of change as the individual fruit, leaf and phloem development in this work, suggesting thatthe four genes may have overlapping, complementary, or cooperative functions in development within a specific tissue. A previous report suggested that five HD-ZIP III family genes may have antagonistic functions in xylem differentiation in Arabidopsis (Du et al., 2015). Further gene transformation needs to be carried out for more comprehensive understanding of the function of miR166 in regulating HD-ZIP III family genes in peach.

\section{Conflicts of interest}

The authors declare no conflicts of interest.

\section{ACKNOWLEDGMENTS}

The authors thank the National Natural Science Foundation of China (\#31401851); Natural Science Foundation of Jiangsu Province (\#BK20130700); earmarked fund of China Agriculture Research System (\#CARS-31); and the Youth Foundation of Jiangsu Academy of Agricultural Sciences (\#ZX(15)4024), for financial support.

\section{REFERENCES}

Arenas-Huertero C, Perez B, Rabanal F, Blanco-Melo D, et al. (2009). Conserved and novel miRNAs in the legume Phaseolus vulgaris in response to stress. Plant Mol. Biol. 70:385-401.

Ariel FD, Manavella PA, Dezar CA and Chan DR (2007). The true story of the HD-ZIP family. Trends Plant Sci. 12:419-426.

Asha S, Nisha J and Soniya EV (2013). In silico characterisation and phylogenetic analysis of two evolutionarily conserved miRNAs (miR166 and miR171) from black pepper (Piper nigrum L.). Plant Mol. Biol. Rep. 31: 707-718.

Barik S, SarkarDas S, Singh A, Gautam V, et al. (2014). Phylogenetic analysis reveals conservation and diversification of microRNA166 genes among diverse plant species. Genomics 103: 114-121.

Bartel DP (2004). MicroRNAs: genomics, biogenesis, mechanism, and function. Cell 116: 281-297.

Bustin SA, Benes V, Garson JA, Hellemans J, et al. (2009). The MIQE guidelines: minimum information for publication of quantitative real-time PCR experiments. Clin. Chem. 55: 611-622. 
Chen X (2004). A microRNA as a translational repressor of APETALA2 in Arabidopsis flower development. Science 303: 2022-2025.

Donaire L, Pedrola L, Rosa RDL and Llave C (2011). High-throughput sequencing of RNAs silencing-associated small RNAs in olive (Olea europaea L.). PLoS One 6: e27916.

Du Q, Avci U, Shengben Li, LinaGallego-Giraldo, et al. (2015). Activation of miR165b represses AtHB15 expression and induces pith secondary wall development in Arabidopsis. Plant J. 83: 388-400.

Eldem V, Okay S and Unver T (2013). Plant microRNAs: new players in functional genomics. Turk. J. Agric. For. 37:1-21.

Emery JF, Floyd SK, Alvarez J, Eshed Y, et al. (2003). Radial patterning of Arabidopsis shoots by class III HD-ZIP and KANADI genes. Curr. Biol. 13:1768-1774.

Floyd SK and Bowman JL (2004). Gene regulation: ancient microRNA target sequences in plants. Nature 428: 485-486.

Javelle M, Klein-Cosson C, Vernoud V, Boltz V, et al. (2011). Genome-wide characterization of the HD-ZIP IV transcription factor family in maize: preferential expression in the epidermis. Plant Physiol. 157: 790-803.

Jiao YP, Song WB, Zhang M and Lai JS (2011). Identification of novel maize miRNAs by measuring the precision of precursor processing. BMC Plant Biol. 11: 141-155.

Jones-Rhoades MW and Bartel DP (2004). Computational identification of plant miRNAs and their targets, including a stressinduced miRNA. Mol. Cell. 14: 787-799.

Ko JH, Prassinos C and Han KH (2006). Developmental and seasonal expression of PtaHB1, a Populus gene encoding a class III HD-ZIP protein, is closely associated with secondary growth and inversely correlated with the level of microRNA (miR166). New Phytol. 169: 469-478.

Livak KJ and Schmittgen TD (2001). Analysis of relative gene expression data using real-time quantitative PCR and the $2^{-\Delta \Delta C t}$ method. Methods 25: 402-408.

Mallory AC, Dugas DV, Bartel DP and Bartel B (2004). MicroRNA regulation of NAC-domain targets is required for proper formation and separation of adjacent embryonic, vegetative, and floral organs. Curr. Biol. 14: 1035-1046.

Mukherjee K and Burglin TR (2006). MEKHLA, a novel domain with similarity to PAS domains is fused to plant HD-ZIP III homeodomain proteins. Plant Physiol. 140: 1142-1150.

Nicolas FE, Pais H, Schwach F, Lindow M, et al. (2008). Experimental identification of microRNA140 targets by silencing and overexpression miR140. RNA 14: 2513-2520.

Ong SS and Wickneswari R (2012). Characterization of microRNAs expressed during secondary wall biosynthesis in Acacia mangium. PloS One 7: e49662.

Palatnik JF, Allen E, Wu X, Schommer C, et al. (2003). Control of leaf morphogenesis by microRNAs. Nature 425: $257-263$.

Park W, Li J, Song R, Messing J, et al. (2002). CARPEL FACTORY, a dicer homolog, and HEN1, a novel protein, act in microRNA metabolism in Arabidopsis thaliana. Curr. Biol. 12: 1484-1495.

Prigge MJ and Clark SE (2006). Evolution of the class III HD-ZIP gene family in land plants. Evol. Devel. 8: $350-361$.

Prigge MJ, Otsuga D, Alonso JM, Ecker JR, et al. (2005). Class III homeodomain-leucine zipper gene family members have overlapping, antagonistic and distinct roles in Arabidopsis development. Plant Cell 17: 61-76.

Qiu CX, Xie FL, Zhu YY, Guo K, et al. (2007). Computational identification of microRNAs and their targets in Gossypiumhirsutum expressed sequence tags. Gene 395: 49-61.

Rhoades M, Reinhart B, Lim L, Burge C, et al. (2002). Prediction of plant microRNA targets. Cell 110: 513-520.

Ruberti I, Sessa G, Lucchetti I and Morelli G (1991). A novel class of plant proteins containing a homeodomain with a closely linked leucine zipper motif. EMBO J. 10: 1787-1791.

Sessa G, Steindler C, Morelli G and Ruberti I (1998). The Arabidopsis Athb-8, -9 and -14 genes are members of a small gene family coding for highly related HD-ZIP proteins. Plant Mol. Biol. 38: 609-622.

Song CN, Jia QD, Fang JG, Li F, et al. (2009). Computational identification of citrus microRNAs and target analysis in citrus expressed sequence tags. Plant Biol. 12: 927-934.

Tong ZG, Gao ZH, Wang F, Zhou J, et al. (2009). Selection of reliable reference genes for gene expression studies in peach using real-time PCR. BMC Mol. Biol. 10:71.

Turner S, Gallois P and Brown D (2007). Tracheary element differentiation. Annu. Rev. Plant Biol. 58: 407-433.

Unver T, Parmaksız I and Du E (2010). Identification of conserved microRNAs and their target transcripts in opium poppy (Papaver somniferum L.). Plant Cell. Rep. 29: 757-769.

Zhang CH, Zhang YP, Guo L, Han J, et al. (2012). Characterization of the miR165 family and its target gene Pp-ATHB8 in Prunus persica. Sci. Hort. 146: 21-28.

Zhang CH, Ma RJ, Shen ZJ, Sun X, et al. (2014). Genome-wide analysis of the homeodomain-leucine zipper (HD-ZIP) gene family in peach (Prunus persica). Genet. Mol. Res. 13: 2654-2668.

Zhao CZ, Xia H, Frazier TP, Yao YY, et al. (2010). Deep sequencing identifies novel and conserved microRNAs in peanuts (Arachis hypogaea L.). Plant Biol. 10:3-15.

Zhong RQ and Zheng HY (2007). Regulation of HD ZIP genes by microRNA165. Plant Signal. Behav. 2: $351-353$. 